\title{
Time Scale and its Application in Perturbation Theory
}

\author{
S. Olszewski \\ Institute of Physical Chemistry, Polish Academy of Sciences, Warsaw, Poland
}

Z. Naturforsch. 46a, 313-320 (1991); received January 7, 1991

A circular scale of time is proposed and applied to the calculation of the Rayleigh-Schrödinger perturbation energy for a non-degenerate state.

\section{Introduction}

In physics, time is - in principle - a coordinate: in a non-relativistic theory this coordinate may vary independently of the spatial coordinates, whereas in a relativistic case the time coordinate is coupled with its spatial counterparts. This situation holds equally for classical and quantum physics. A scale of time is then assumed in which the time coordinate may vary from a minus infinity called the "past" to a plus infinity called the "future". A time scale of this kind we call linear, see Figure 1.

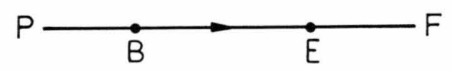

Fig. 1. Linear time scale. $\mathrm{P}$-past, $\mathrm{F}$-future, $\mathrm{B}, \mathrm{E}$ - beginning and end points on the scale.

One of the applications of a time scale is perturbation theory. In reality, the perturbation calculation based on a linear scale of time is quite complicated: we construct the time-dependent vacuum amplitude for a given perturbation order, then calculate its logarithm and differentiate with respect to time, see e.g. [1]. Going with the time variable, corrected by an imaginary infinitesimal part, to infinity we find all the components of the perturbation series. The procedure is not straightforward, especially because there exists a diagrammatical representation for the vacuum amplitude but not for the limit of the logarithmic time derivative, exhibiting some lack in the diagrammatical representation for the individual terms of the perturbation series.

The purpose of the present paper is to indicate that a time scale which is different from linear may be - at

Reprint requests to Prof. Dr. S. Olszewski, Instytut Chemii Fizycznej P.A.N., ul. Kasprzaka 44/52, 01-224 Warszawa, Poland.

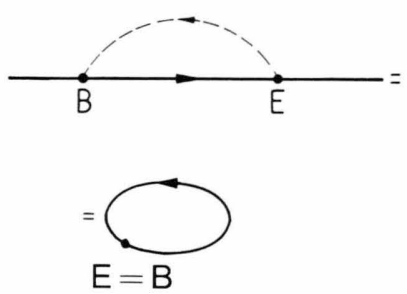

Fig. 2. Formation of a circular time scale. B and E-beginning and end points on the scale - merge.

least in some cases - also of use. This is done for the perturbation calculation of a non-degenerate and non-relativistic quantum-mechanical energy state. The applied scale is topologically equivalent to a circle, which means that the end point and the beginning point on the scale merge, see Figure 2. A scale of this kind may occur also to be more natural for some classical systems, for example a perfect harmonic oscillator: since there is no physical parameter in such an oscillator which enables us to distinguish between one and another oscillation periods, the same should apply to the time variable.

\section{Rayleigh-Schrödinger One-Particle Perturbation Theory}

The non-relativistic Rayleigh-Schrödinger (RS) perturbation theory can be useful even to a high perturbation order, see e.g. [2]. As mentioned above, the perturbation energy $\Delta E$ of a one-particle system in state $n$ can be represented with the aid of the timedependent quantum vacuum amplitude $R(t)$ for this system [1]. This amplitude is the sum of probability amplitudes for all various ways, the system can start from its initial (unperturbed) state, scatter on a perturbation potential $V(\boldsymbol{r})$ zero, one, or more times and return to the unperturbed state. The linked-cluster

0932-0784/91/0400-0313 \$ 01.30/0. - Please order a reprint rather than making your own copy. 
theorem gives

$$
\begin{aligned}
\Delta E & =\lim _{t \rightarrow \infty(1-i \eta)} i \frac{\mathrm{d}}{\mathrm{d} t} \ln R(t) \\
& =\lim _{t \rightarrow \infty(1-i \eta)} i \frac{\mathrm{d}}{\mathrm{d} t}\left\{(-1) V_{n n} \int_{0}^{t} \mathrm{~d} t_{1} i G_{0}^{-}\left(n, t_{1}-t_{1}\right)+\sum_{p \neq n}(-1)^{2} V_{n p} V_{p n} \int_{0}^{t} \mathrm{~d} t_{1} \int_{0}^{t} \mathrm{~d} t_{2} i G_{0}^{+}\left(p, t_{2}-t_{1}\right) i G_{0}^{-}\left(n, t_{1}-t_{2}\right)\right.
\end{aligned}
$$

where $\eta$ is an infinitesimal, yet positive number; for an unperturbed energy state $E_{k}$ we have

$$
\begin{aligned}
G_{0}^{-}\left(k, t_{2}-t_{1}\right) & =i \Theta_{t_{1}-t_{2}} \exp \left\{-i E_{k}\left(t_{2}-t_{1}\right)\right\}, \\
& =i \quad \text { for } t_{2}=t_{1} ; \\
G_{0}^{+}\left(k, t_{2}-t_{1}\right) & =-i \Theta_{t_{2}-t_{1}} \exp \left\{-i E_{k}\left(t_{2}-t_{1}\right)\right\}, \\
& =0 \text { for } t_{2}=t_{1} ; \\
\Theta_{t_{2}-t_{1}} & =1 \quad \text { if } \quad t_{2}>t_{1}, \\
& =0 \text { if } \quad t_{2}<t_{1},
\end{aligned}
$$

$V_{n n}, V_{n p}, \ldots$ are the matrix elements between the unperturbed one-particle wave functions and the perturbation potential. A graphical representation of (1) is

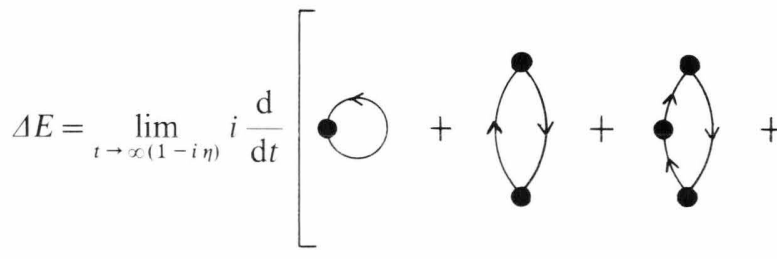

where in the square brackets we have the 1st, 2 nd, $3 \mathrm{rd}, 4$ th ... order diagrams. The representation corresponds to a sequence of scattering events $t_{1}, t_{2}, t_{3}, t_{4} \ldots$ which can be arranged, however, in different ways. For example, for a fourth perturbation order, the scattering events - beyond $t_{1}$ - can be arranged as

$$
\begin{aligned}
& t_{2}, t_{3}, t_{4}, \\
& t_{2}, t_{4}, t_{3}, \\
& t_{3}, t_{4}, t_{2}, \\
& t_{4}, t_{3}, t_{2}, \\
& t_{4}, t_{2}, t_{3}, \\
& t_{3}, t_{2}, t_{4},
\end{aligned}
$$

which exhausts all linked diagrams of order 4 which enter representation (5). In effect, the number $L_{N}$ of the linked diagrams of order $N$ entering (5) is

$$
L_{N}=(N-1) !
$$

This is not a satisfactory situation since, in fact, the number of terms which are given, for example, by the Brueckner-Huby bracketing technique [3, 4] and enter the RS expression for $\Delta E$ of order $N$ is given by [5]

$$
S_{N}=\frac{(2 N-2) !}{N !(N-1) !}
$$

leading evidently to

$$
S_{N} \neq L_{N}
$$

for any $N>3$.

The purpose and procedure of the next sections is twofold. On the first step we show that $\Delta E$ can be obtained on the basis of a single matrix element for a perturbation potential under the condition that we

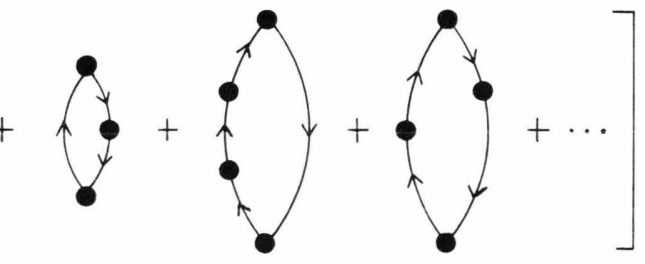

apply some postulates concerning the sequence of scattering events on a circular time scale. On the second step we show for this scale that the same combination of the scattering events which leds to $\Delta E$ gives also corresponding diagrams for the RS terms.

\section{Time Scale and the Main Term of the RS Theory}

Similarly to Sect. 2 we assume the $N$-th order perturbation to be connected with $N$ time intervals

$$
\begin{aligned}
& \left\{t^{\prime}\right\}: \quad t_{(01)}<t^{\prime}<t_{(1)}, \quad n \rightarrow p, \\
& \left\{t^{\prime \prime}\right\}: \quad t_{(02)}<t^{\prime \prime}<t_{(2)}, \quad p \rightarrow q, \\
& \left\{t^{(N)}\right\}: \quad t_{(O N)}<t^{(N)}<t_{(N)}, \quad z \rightarrow n
\end{aligned}
$$

during which a system from its initial state $n$ comes back to this state. During the first interval $n$ is scattered to $p$, during the second one $p$ is scattered to $q$, in 
the last interval $z$ is scattered back to $n$; for $N=1$ we have obviously the $n \rightarrow n$ scattering. [The superscripts of $t$ in (10) for time intervals further than the third one will be written in Roman style.] We postulate that a part of $\Delta E$ which correspods to $N$, called $\Delta E^{(N)}$, is

$$
\Delta E^{(N)}=\sum_{c} \Delta E_{c}^{(N)},
$$

where the sum is extended over all allowed sequence (cyles) of the time intervals for a given $N$. Any $\Delta E_{c}^{(N)}$ contributed by a cycle is obtained from the equation

$\Delta E_{c}^{(N)} i \tau_{(N)}=S_{c} \int \mathrm{d} \boldsymbol{r} \int i \mathrm{~d} t \psi_{\alpha}^{*}(\boldsymbol{r}, t) \hat{V}(\boldsymbol{r}) \psi_{\beta}(\boldsymbol{r}, t)$,

where $\tau_{(N)}$ is the length of the last time interval in a cycle,

$$
\begin{aligned}
& \psi_{\alpha}(\boldsymbol{r}, t)=\exp \left\{-i E_{\alpha} t\right\} \varphi_{\alpha}(\boldsymbol{r}), \\
& \psi_{\beta}(\boldsymbol{r}, t)=\exp \left\{-i E_{\beta} t\right\} \varphi_{\beta}(\boldsymbol{r}),
\end{aligned}
$$

$(\hbar=1)$ and the symbol S means that integrations have to be performed in (11) over all time intervals in a

$$
\begin{aligned}
& U_{n p} \frac{1}{E_{n}-E_{p}} \int \mathrm{d} \boldsymbol{r}^{\prime \prime}{ }^{t^{\prime \prime \prime}} \int_{t_{(02)}}^{t_{(2)}} \mathrm{i} \mathrm{d} t^{\prime \prime} \exp \left\{i E_{p} t^{\prime \prime}\right\} \varphi_{p}^{*}\left(\boldsymbol{r}^{\prime \prime}\right) V\left(\boldsymbol{r}^{\prime \prime}\right) \exp \left\{-i E_{q} t^{\prime \prime}\right\} \varphi_{q}\left(\boldsymbol{r}^{\prime \prime}\right) \exp \left\{i\left(E_{n}-E_{p}\right) t^{\prime \prime}\right\} \\
& \quad=U_{n p} \frac{1}{E_{n}-E_{p}} U_{p q} \frac{1}{E_{n}-E_{q}} \exp \left\{i\left(E_{n}-E_{q}\right) t^{\prime \prime}\right\} \begin{array}{l}
t^{\prime \prime \prime}=t_{(2)} \\
t^{\prime \prime}=t_{(02)}
\end{array}=U_{n p} \frac{1}{E_{n}-E_{p}} U_{p q} \frac{1}{E_{n}-E_{q}} \exp \left\{i\left(E_{n}-E_{q}\right) t_{(2)}\right\}
\end{aligned}
$$

on the condition that the reasoning concerning the limits of $\left\{t^{\prime}\right\}$ presented below (15) is applied also in the case of the limits of $\left\{t^{\prime \prime}\right\}$. The calculation procedure can be extended to an arbitrary number of intervals (10) accompanied by the integration done over the corresponding space variable and the potential (13). In case $\left\{t^{\prime \prime \prime}\right\}$ is a last interval in a cycle we obtain as total result of the integration over $\left\{t^{\prime}\right\},\left\{t^{\prime \prime}\right\},\left\{t^{\prime \prime \prime}\right\}$ and those over $\boldsymbol{r}^{\prime}, \boldsymbol{r}^{\prime \prime}, \boldsymbol{r}^{\prime \prime \prime}$ :

$$
\begin{aligned}
& U_{n p} \frac{1}{E_{n}-E_{p}} U_{p q} \frac{1}{E_{n}-E_{q}} \int \mathrm{d} \boldsymbol{r}^{\prime \prime \prime} \int_{t^{\prime \prime \prime}=t_{(03)}{ }^{\prime \prime \prime}=t_{(3)}} \mathrm{i} \mathrm{d} t^{\prime \prime \prime} \\
& \cdot \exp \left\{i E_{q} t^{\prime \prime \prime}\right\} \varphi_{q}^{*}\left(\boldsymbol{r}^{\prime \prime \prime}\right) V\left(\boldsymbol{r}^{\prime \prime \prime}\right) \\
& \cdot \exp \left\{-i E_{n} t^{\prime \prime \prime}\right\} \varphi_{n}\left(\boldsymbol{r}^{\prime \prime \prime}\right) \exp \left\{i\left(E_{n}-E_{q}\right) t^{\prime \prime \prime}\right\} \\
& =U_{n p} \frac{1}{E_{n}-E_{p}} U_{p q} \frac{1}{E_{n}-E_{q}} U_{q n} i \tau_{(3)},
\end{aligned}
$$

where - similarly to (16) - we have put under the integral

$$
t_{(2)} \equiv t^{\prime \prime \prime}
$$


Expression (18) substituted into (11) gives $\Delta E_{c}^{(3)}=\Delta E_{m}^{(3)}$, which we call the main term of $\Delta E^{(3)}$. Similarly $\Delta E_{m}^{(N)}$ can be obtained for any $N$ in the way indicated above, which means that the upper limit $t_{(v-1)}$ of an interval $\left\{t^{(v-1)}\right\}$ enters the next interval $\left\{t^{(v)}\right\}$ as a variable $t^{(v)}$.

\section{Elimination principle for equal times}

Our purpose is now to get the remainder of $S_{N}-1$ terms $\Delta E_{c}^{(N)}$ which enter $\Delta E^{(N)}$ beyond $\Delta E_{m}^{(N)}$. A guide to find the cycles for the components of the peturbation series other than $\Delta E_{m}^{(N)}$ is the elimination principle. In the calculations of Sect. 3, (10), we assumed tacitly that the time variable in any interval is larger than in a preceding interval:

$$
\left\{t^{\prime}\right\}<\left\{t^{\prime \prime}\right\}<\left\{t^{\prime \prime \prime}\right\}<\ldots<\left\{t^{(N-1)}\right\}<\left\{t^{(N)}\right\} .
$$

The elimination principle assumes that the sequence given in (20) - except for the last inequality

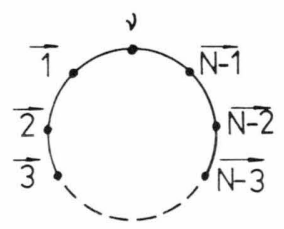

Fig. 3. Circular scale with $N$ time intervals on it; $v$ represents the beginning-end (BE) point on the scale.
$\left\{t^{(N-1)}\right\}<\left\{t^{(N)}\right\}$ - may be violated. For example, for $N=4$ we may have the following time contractions:

$$
\begin{aligned}
& \left\{t^{\prime}\right\}=\left\{t^{\prime \prime}\right\}, \\
& \left\{t^{\prime \prime}\right\}=\left\{t^{\prime \prime \prime}\right\}, \\
& \left\{t^{\prime}\right\}=\left\{t^{\prime \prime \prime}\right\},
\end{aligned}
$$

and

$$
\left\{t^{\prime}\right\}=\left\{t^{\prime \prime}\right\}=\left\{t^{\prime \prime \prime}\right\} .
$$

We shall demonstrate that any of the allowed contractions represent a term of the RS series; in effect, for $N=4$ the contractions (21)-(21 c) together with the main term represented by the sequence

$$
\left\{t^{\prime}\right\}<\left\{t^{\prime \prime}\right\}<\left\{t^{\prime \prime \prime}\right\}<\left\{t^{\mathrm{IV}}\right\}
$$

(and calculated according to Sec. 3) give the total number

$S_{4}=\frac{(2 * 4-2) !}{4 !(4-1)}=\frac{6 !}{4 ! 3 !}=5$

of the perturbation terms of order 4 . The allowed contractions can be chosen according to the following topological rule. Let us set consecutively $N$ time intervals on a circular scale of time beginning from some point $v$ which is also the end point on the scale; see Figure 3. If all $N-1$ points beyond $v$ representing the interval ends are projected to any other point different from $v$ in the way that projection lines do not cross we

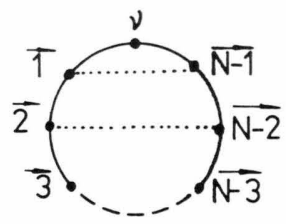

Fig. 4 .

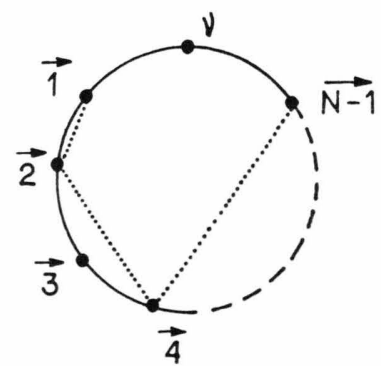

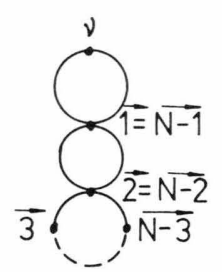

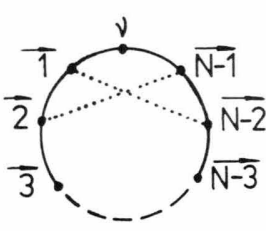

Fig. 6. Example of the forbidden contraction on a circular time scale.

Fig. 5.

Fig. 4 and 5. Examples of the allowed contractions on a circular time scale. 
obtain the time contractions for all terms of the RS series of order $N$. The examples of corresponding allowed diagrams are represented in Figs. 4 and 5; an example of a forbidden diagram is represented on Figure 6. In the present Section we show how contractions may give the perturbation terms. First we postulate that a contraction between two intervals which have the end points say $p$ and $q$, see (10), leads to an equality

$$
\exp \left\{-i E_{p} t^{\prime}\right\} \varphi_{p}\left(\boldsymbol{r}^{\prime}\right)=\exp \left\{-i E_{q} t^{\prime \prime}\right\} \varphi_{q}\left(\boldsymbol{r}^{\prime \prime}\right)
$$

for any $t^{\prime}, t^{\prime \prime}, \boldsymbol{r}^{\prime}$, and $\boldsymbol{r}^{\prime \prime}$, so

$$
E_{p}=E_{q}
$$

a similar contraction between $p$ and $r$ would mean

$$
\begin{aligned}
& \exp \left\{-i E_{p} t^{\prime}\right\} \varphi_{p}\left(\boldsymbol{r}^{\prime}\right)=\exp \left\{-i E_{\boldsymbol{r}} t^{\prime \prime \prime}\right\} \varphi_{\boldsymbol{r}}\left(\boldsymbol{r}^{\prime \prime \prime}\right) \\
& E_{p}=E_{\boldsymbol{r}}
\end{aligned}
$$

etc. We apply (23) -(23a) and (23)-(24a), respectively, in the calculation of the RS terms represented by (21) and (21 b). The first interval of time gives, see (14) and inferences below (15),

$$
U_{n p}\left(E_{n}-E_{p}\right)^{-1} \exp \left\{i\left(E_{n}-E_{p}\right) t_{(1)}\right\},
$$

so contraction (21) together with (23) and (23a) gives during the second interval

$$
\begin{aligned}
& U_{n p}\left(E_{n}-E_{p}\right)^{-1} \int \mathrm{d}\left(\boldsymbol{r}^{\prime}=\boldsymbol{r}^{\prime \prime}\right) \int_{t^{\prime}=t^{\prime \prime}=t_{(01)}=t_{(02)}}^{t^{\prime}=t^{\prime \prime}=t_{(1)}=t_{(2)}} i \mathrm{~d}\left(t^{\prime}=t^{\prime \prime}\right) \\
& \cdot \exp \left\{i E_{p}\left(t^{\prime}=t^{\prime \prime}\right)\right\} \varphi_{p}^{*}\left(\boldsymbol{r}^{\prime}=\boldsymbol{r}^{\prime \prime}\right) V\left(\boldsymbol{r}^{\prime}=\boldsymbol{r}^{\prime \prime}\right) \\
& \cdot \exp \left\{-i\left(E_{q}=E_{p}\right)\left(t^{\prime}=t^{\prime \prime}\right)\right\} \varphi_{q=p}\left(\boldsymbol{r}^{\prime}=\boldsymbol{r}^{\prime \prime}\right) \\
& \cdot \exp \left\{i\left(E_{n}-E_{p}\right)\left(t^{\prime}=t^{\prime \prime}\right)\right\} \\
&= U_{n p}\left(E_{n}-E_{p}\right)^{-1} U_{p p}\left(E_{n}-E_{p}\right)^{-1} \\
& \cdot \exp \left\{i\left(E_{n}-E_{p}\right)\left(t_{(1)}=t_{(2)}\right)\right\}
\end{aligned}
$$

the last term under the integral comes from (14a), (16), and (21). On the other hand, in order to make use of the contractions (21 a) and ( 21 b) we take - on the first step - the result (17) of the integrations done over $\left\{t^{\prime}\right\}$, $\left\{t^{\prime \prime}\right\}$ :

$U_{n p} \frac{1}{E_{n}-E_{p}} U_{p q} \frac{1}{E_{n}-E_{q}} \exp \left\{i\left(E_{n}-E_{q}\right) t_{(2)}\right\}$

and next apply equalities of the kind given in (23)(24a). For the case of (21a) we obtain

$$
\begin{aligned}
& U_{n p} \frac{1}{E_{n}-E_{p}} U_{p q} \frac{1}{E_{n}-E_{q}} \int \mathrm{d}\left(\boldsymbol{r}^{\prime \prime}=\boldsymbol{r}^{\prime \prime \prime}\right) \prod_{t^{\prime \prime}=t^{\prime \prime \prime}=t_{(02)}=t_{(03)}}^{t^{\prime \prime}=t^{\prime \prime \prime}=t_{(2)}=t_{(3)}} i \mathrm{~d}\left(t^{\prime \prime}=t^{\prime \prime \prime}\right) \exp \left\{i E_{q}\left(t^{\prime \prime}=t^{\prime \prime \prime}\right)\right\} \varphi_{q}^{*}\left(\boldsymbol{r}^{\prime \prime}=\boldsymbol{r}^{\prime \prime \prime}\right) V\left(\boldsymbol{r}^{\prime \prime}=\boldsymbol{r}^{\prime \prime \prime}\right) \\
& \cdot \exp \left\{-i\left(E_{\boldsymbol{r}}=E_{q}\right)\left(t^{\prime \prime}=t^{\prime \prime \prime}\right)\right\} \varphi_{\boldsymbol{r}=q}\left(\boldsymbol{r}^{\prime \prime}=\boldsymbol{r}^{\prime \prime \prime}\right) \exp \left\{i\left(E_{n}-E_{q}\right)\left(t^{\prime \prime}=t^{\prime \prime \prime}\right)\right\}=U_{n p} \frac{1}{E_{n}-E_{p}} U_{p q} \frac{1}{E_{n}-E_{q}} U_{q q} \frac{1}{E_{n}-E_{q}} \\
& \cdot \exp \left\{i\left(E_{n}-E_{q}\right)\left(t_{(2)}=t_{(3)}\right)\right\},
\end{aligned}
$$

whereas for the case of $(21 \mathrm{~b})$ we have

$$
\begin{aligned}
& U_{n p} \frac{1}{E_{n}-E_{p}} U_{p q} \frac{1}{E_{n}-E_{q}} \int \mathrm{d}\left(\boldsymbol{r}^{\prime}=\boldsymbol{r}^{\prime \prime \prime}\right) \int_{t^{\prime}=t^{\prime \prime \prime}=t_{(01)}=t_{(03)}}^{t^{\prime}=t^{\prime \prime \prime}=t_{(1)}=t_{(3)}} i \mathrm{~d}\left(t^{\prime}=t^{\prime \prime \prime}\right) \exp \left\{i E_{q}\left(t^{\prime}=t^{\prime \prime \prime}\right)\right\} \varphi_{q}^{*}\left(\boldsymbol{r}^{\prime}=\boldsymbol{r}^{\prime \prime \prime}\right) V\left(\boldsymbol{r}^{\prime}=\boldsymbol{r}^{\prime \prime \prime}\right) \\
& \cdot \exp \left\{-i\left(E_{\boldsymbol{r}}=E_{p}\right)\left(t^{\prime}=t^{\prime \prime \prime}\right)\right\} \varphi_{\boldsymbol{r}=p}\left(\boldsymbol{r}^{\prime}=\boldsymbol{r}^{\prime \prime \prime}\right) \exp \left\{i\left(E_{n}-E_{q}\right)\left(t^{\prime}=t^{\prime \prime \prime}\right)\right\}=U_{n p} \frac{1}{E_{n}-E_{p}} U_{p q} \frac{1}{E_{n}-E_{q}} U_{q p} \frac{1}{E_{n}-E_{p}} \\
& \cdot \exp \left\{i\left(E_{n}-E_{p}\right)\left(t_{(1)}=t_{(3)}\right)\right\} .
\end{aligned}
$$

In case of the contraction (21 c) we apply - on the first step - the result for (21) given in (25). We have

$$
\begin{aligned}
U_{n p}\left(E_{n}-\right. & \left.E_{p}\right)^{-2} U_{p p} \int \mathrm{d}\left(\boldsymbol{r}^{\prime}=\boldsymbol{r}^{\prime \prime \prime}\right) \int_{t^{\prime}=t^{\prime \prime \prime}=t_{(01)}=t_{(03)}}^{t^{\prime}=t^{\prime \prime \prime}=t_{(1)}=t_{(3)}} i \mathrm{~d}\left(t^{\prime}=t^{\prime \prime \prime}\right) \cdot \exp \left\{i\left(E_{q}=E_{p}\right)\left(t^{\prime}=t^{\prime \prime \prime}\right)\right\} \varphi_{q=p}^{*}\left(\boldsymbol{r}^{\prime} \equiv \boldsymbol{r}^{\prime \prime \prime}\right) V\left(\boldsymbol{r}^{\prime}=\boldsymbol{r}^{\prime \prime \prime}\right) \\
\cdot & \exp \left\{-i\left(E_{\boldsymbol{r}}=E_{p}\right)\left(t^{\prime}=t^{\prime \prime \prime}\right)\right\} \varphi_{\boldsymbol{r}=p}\left(\boldsymbol{r}^{\prime}=\boldsymbol{r}^{\prime \prime \prime}\right) \cdot \exp \left\{i\left(E_{n}-E_{p}\right)\left(t^{\prime}=t^{\prime \prime \prime}\right)\right\} \\
= & U_{n p}\left(E_{n}-E_{p}\right)^{-3} U_{p p} U_{p p} \exp \left\{i\left(E_{n}-E_{p}\right)\left(t_{(1)}=t_{(3)}\right)\right\} .
\end{aligned}
$$


PERTURBATION ORDER:1

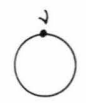

PERTURBATION ORDER:2<smiles>CC1CCCCCC1</smiles>

PERTURBATION ORDER:3<smiles>C1CCCCCC1</smiles><smiles>CC1CCCCCC1</smiles>

$\mathrm{a}_{3}$ $I_{3}$

PERTURBATION ORDER 4<smiles>CC1CCCCCCC1</smiles><smiles>[Y2][C]1CCCCCCCCCCCC1</smiles><smiles>CC1CCC2CCCCC2C1</smiles><smiles>C1CCCCCC1</smiles>

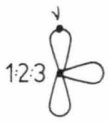

$\mathrm{III}_{4}$<smiles>CC1CCCCCC1</smiles><smiles>[10BH2]</smiles><smiles>C1CCC2(CC1)CCCCS2</smiles>

$1 \mathrm{II}_{6}$<smiles></smiles>

$\mathrm{VI}_{6}$<smiles>CCC12CCCCCCC1(CC)CCC2</smiles>

$1 X_{6}$

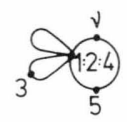

$\mathrm{XII}$

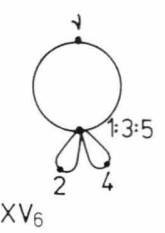<smiles>CC1(C)CCCCCCC1(C)C</smiles>

$\mathrm{XVIII} \mathrm{I}_{6}$<smiles>O=C1CCCCCCC12CO2</smiles>

$X X I_{6}$<smiles>CC1CCCCCCC1</smiles><smiles>[Z2]C1CCCCC12CCCCS2</smiles>

$\mathrm{IV}_{6}$

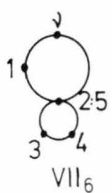<smiles>[Y6]C1CCCCCC1(C)C</smiles><smiles>CC1(C)CCCSCC1</smiles><smiles>CC1(C)CC2CCC1CC2</smiles>

$X \mathrm{II}_{6}$<smiles>c1c[14cH][14cH][14cH][14cH][14cH][14cH][cH-]1</smiles>

$\mathrm{VIII}_{6}$<smiles>CC1CCCCCC1C</smiles>

$0_{5}$<smiles>CC1(C)CCCCC12CCCCC2</smiles><smiles></smiles><smiles>[Y5][C@H]1C[14CH2][14CH2][14CH2][14CH2][14CH2]1</smiles>

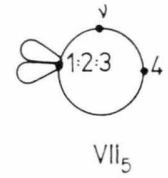

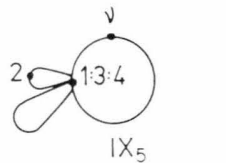<smiles>[Y9]C1([As])CCCCCCC1C</smiles>

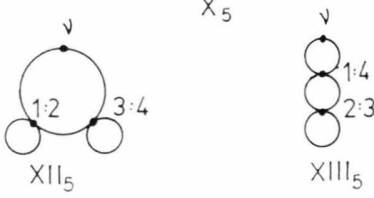<smiles>CC1CCCCCCCCCCCC1</smiles>

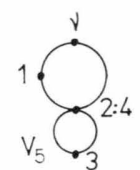

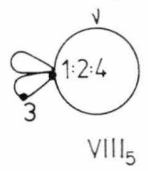

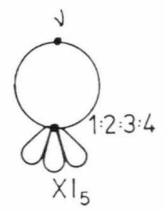

$X I_{5}$<smiles>[Y6]C1([Z6])CCCCCCC1(C)S</smiles><smiles>CC1(CO)SCCCCCC1=O</smiles>

$\mathrm{XVII}{ }_{6}$

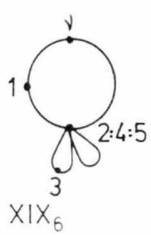

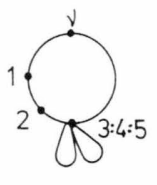

$x x_{6}$

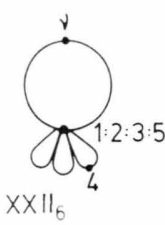

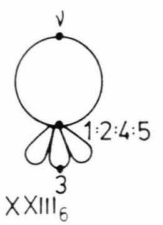

Fig. 7. Diagrams for the perturbation orders from $N=1$ to $N=6$; the beginning-end point is represented by $1:$; arrows above the point symbols are omitted; the contractions on the scale are represented by colons. The diagram for the main term of order $N$ is labeled by $\mathrm{O}_{N}$. 

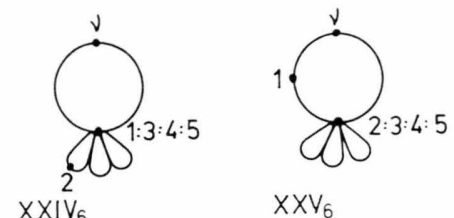

$X X Y_{6}$

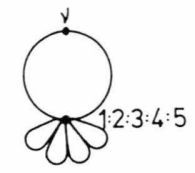

$\mathrm{XXVI} I_{6}$

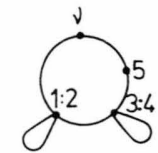

$\mathrm{XXVII} 6$
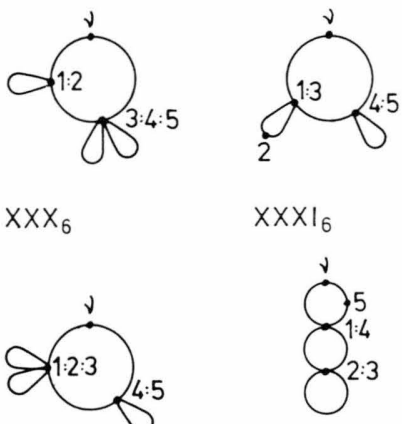

$X X X_{1}$
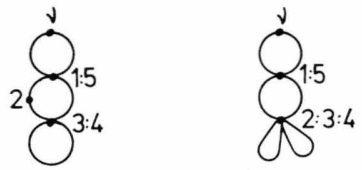

$X X X V I_{6}$

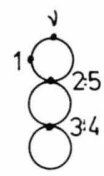

$\mathrm{XXX} \mathrm{XX}_{6}$

Fig. 7.

where again in the last term under the integral the substitution (19) is taken into account together with (21 c).

In order to get the integral over the whole cycle, expression (25) has to be integrated over $t^{\prime \prime \prime}, \boldsymbol{r}^{\prime \prime \prime}$ and $t^{\mathrm{IV}}$, $\boldsymbol{r}^{\mathrm{IV}}$, whereas (26), (27) and (28) solely over $t^{\mathrm{IV}}, \boldsymbol{r}^{\mathrm{IV}}$. This is done according to the rules given in Section 3. We obtain from $(25)$

$$
\begin{gathered}
U_{n p}\left(E_{n}-E_{p}\right)^{-1} U_{p p}\left(E_{n}-E_{p}\right)^{-1} \\
\cdot U_{p r}\left(E_{n}-E_{r}\right)^{-1} U_{r n} i \tau_{(4)} ;
\end{gathered}
$$

from (26):

$$
\begin{gathered}
U_{n p}\left(E_{n}-E_{p}\right)^{-1} U_{p q}\left(E_{n}-E_{q}\right)^{-1} \\
\cdot U_{q q}\left(E_{n}-E_{q}\right)^{-1} U_{q n} i \tau_{(4)}
\end{gathered}
$$

from (27):

$$
\begin{gathered}
U_{n p}\left(E_{n}-E_{p}\right)^{-1} U_{p q}\left(E_{n}-E_{q}\right)^{-1} \\
\cdot U_{q p}\left(E_{n}-E_{p}\right)^{-1} U_{p n} i \tau_{(4)}
\end{gathered}
$$

and from (28):

$$
U_{n p}\left(E_{n}-E_{p}\right)^{-3} U_{p p} U_{p p} U_{p n} i \tau_{(4)} .
$$

\section{Postulate concerning the static part and the sign of $\Delta E_{c}^{(N)}$}

The static part of $\Delta E_{c}^{(N)}$ is essentially a product of the matrix elements $U_{\alpha \beta}$, the number of which is equal to $N$. For example, the static part of the main term for $N=4$ is

$$
U_{n p} U_{p q} U_{q r} U_{r n}
$$

see Section 3. Time contractions make that some indices entering the main static part given in (33) repeat more than twice whereas other indices are cancelled. For example the time contractions (21), (21a), (21 b), and (21 c) discussed in Sect. 4 give respectively

$$
\begin{aligned}
& U_{n p} U_{p p} U_{p r} U_{r n}, \\
& U_{n p} U_{p q} U_{q q} U_{q n}, \\
& U_{n p} U_{p q} U_{q p} U_{p n}, \\
& U_{n p} U_{p p} U_{p p} U_{p n} .
\end{aligned}
$$

Any contraction gives a loop, or loops, on a diagram representing a given cycle of time intervals, and we postulate that the static part corresponding to any loop should be: (i) independent of the position of this loop in a cycle; (ii) equal to the static part of the same loop when it represents a separate cycle. For example, $U_{p p}$ in (34) comes from the loop with only one point on the loop, and we postulate that this static part should be equal to $U_{n n}$, which is a static part for the loop of the same kind making a cycle for its own; the same concerns the loop giving $U_{q q}(34 \mathrm{a})$, and two loops giving $U_{p p} U_{p p},(34 \mathrm{c})$. On the next step the product, $U_{p q} U_{q p}$ in $(34 \mathrm{~b})$ comes from a loop which has two 
Table 1. The correspondence between the diagrams of Fig. 7 and the Brueckner-Huby terms for the RS perturbation series for energy of a non-degenerate state. Perturbation orders from $N=1$ to $N=6$ are examined. The points on diagrams in which $2,3,4 \ldots$ loops touch give, respectively, $\mathrm{P}^{2}, \mathrm{P}^{3}, \mathrm{P}^{4}, \ldots$ and the arcs on the loops between the points are represented by $\mathrm{V}$.

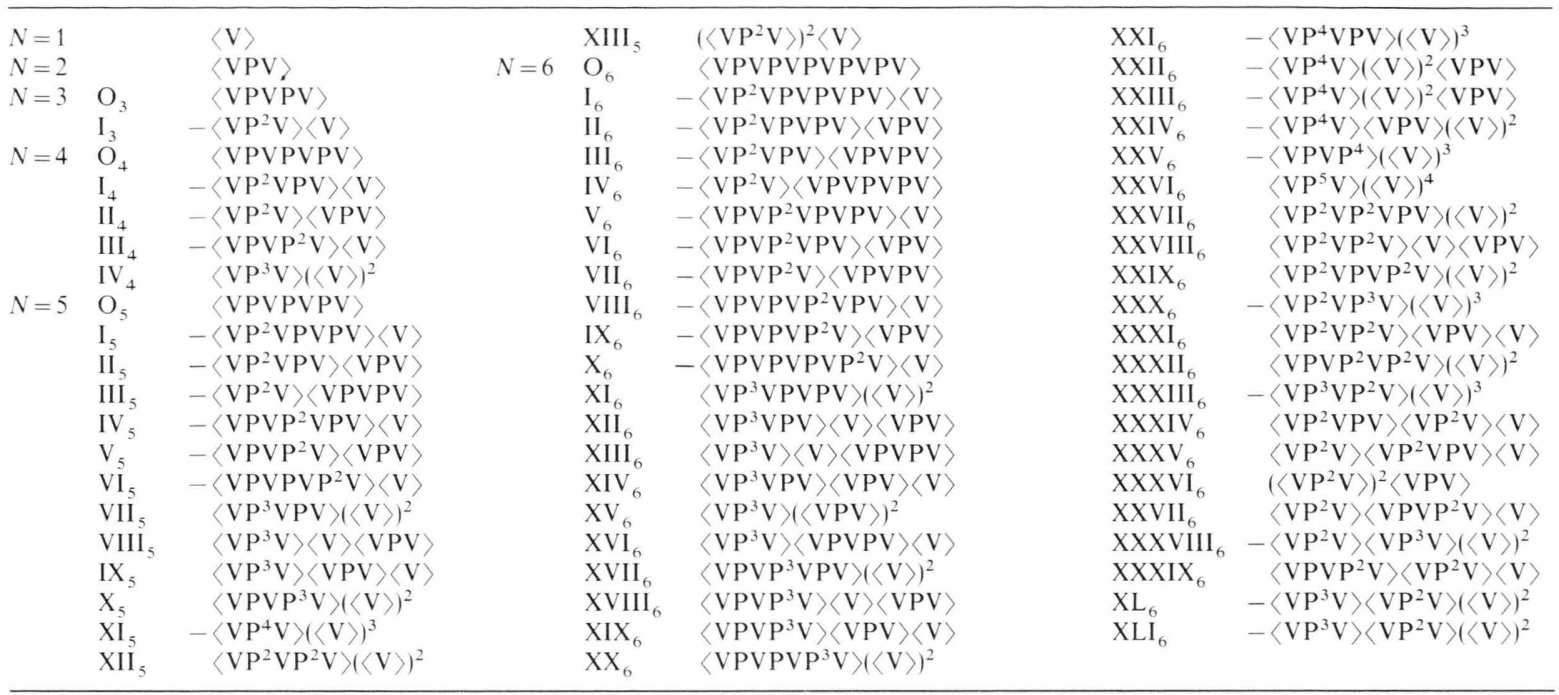

points on it. The static part for this loop - when it represents a separate cycle - is $U_{n q} U_{q n}$, and we postulate that this value should be substituted for $U_{p q} U_{q p}$. In effect, instead of (34)-(34c) we obtain

$$
\begin{aligned}
& U_{n p} U_{p r} U_{r n} U_{n n}, \\
& U_{n p} U_{p q} U_{q n} U_{n n}, \\
& U_{n p} U_{p n} U_{n p} U_{p n}, \\
& U_{n p} U_{p n} U_{n n} U_{n n} .
\end{aligned}
$$

where any term, or product of terms, which begins and ends with the subscript $n$ corresponds to one loop. This procedure can be extended to any cycle. For example, for $N=6$ we have one (main) cycle represented by one loop and 41 cycles having diagrams composed of more than one loop. The diagrams for $N=1,2, \ldots, 6$ are represented on Figure 7 . With the aid of the postulate given above any of the diagrams give some $\Delta E_{c}^{(N)}$ which is equal to some BruecknerHuby $(\mathrm{BH})$ term. A full list representing the correspondence between diagrams and the $\mathrm{BH}$ terms for $N=1,2, \ldots, 6$ is given in Table 1 . Any bracket

$$
\left\langle\mathrm{VP}^{\alpha} \mathrm{VP}^{\beta} \mathrm{VP}^{\gamma} \ldots \mathrm{V}\right\rangle
$$

[1] R. D. Mattuck, A Guide to Feymann Diagrams in the Many-Body Problem, McGraw-Hill, New York 1967.

[2] C. W. Scherr and R. E. Knight, Revs. Mod. Phys. 35, 436 (1963). represents one loop. The sequence VVV ... V within the bracket is an abbreviated formula for

$$
U_{n p} U_{p q} U_{q r} \ldots U_{z n},
$$

whereas the sequence $\mathrm{P}^{\alpha} \mathrm{P}^{\beta} \mathrm{P}^{\gamma} \ldots$ is for

$$
\left(E_{n}-E_{p}\right)^{-x}\left(E_{n}-E_{q}\right)^{-\beta}\left(E_{n}-E_{r}\right)^{-\gamma} \ldots
$$

In a final result for the RS series any sum over $p, q$, $r \ldots$ is done separately for each loop. The sign of $\Delta E_{c}^{(N)}$ is defined by the number $l$ of loops in the diagram by the formula

$$
(-1)^{l+1}
$$

This formula completes the representation of the RS series with the aid of $\Delta E_{c}^{(N)}$.

\section{Acknowledgement}

I am grateful to T. Kwiatkowski for his assistance in preparing the manuscript and to Dr. P. Modrak for his comments.

[3] K. A. Brueckner, Phys. Rev. 100, 361 (1955).

[4] R. Huby. Proc. Phys. Soc. London 78, 529 (1961).

[5] B. Y. Tong. Proc. Phys. Soc. London 80, 1101 (1962). 